10. Hourioux C, Ait-Goughoulte M, Patient R, et al. Core protein domains involved in hepatitis $C$ virus-like particle assembly and budding at the endosplasmic reticulum membrane. Cell Microbiol 2007 ; 9: 1014-27.

11. Ait-Goughoulte M, Hourioux C, Patient R, et al. Core protein cleavage by signal peptide peptidase is required for hepatitis C virus-like particle assembly. J Gen Virol $2006 ; 87: 855-60$.
12. Rouillé $Y$, Helle F, Delgrange D, et al. Subcellular localization of hepatitis $C$ virus structural proteins in a cell culture system that efficiently replicates the virus. J Virol $2006 ; 80: 2832-41$.

13. Fartoux F, Chazouillères 0 , Wendum $D$, et al. Impact of steatosis on progression of fibrosis in patients with mold hepatitis C. Hepatology $2005 ; 41: 82-7$.

14. McLauchlan. Properties of the hepatitis $C$ virus core protein: a srtuctural protein that modulates cellular processes. J Viral Hepat 2000 ; $7: 2-14$.

15. Asselah T, Rubbia-Brandt L, Marcellin P, Negro F. Steatosis in chronic hepatitis C: what does it really matter? Gut 2006; 55 : 123-30.

16. Hourioux C, Patient R, Morin A, et al. The genotype 3 -specific hepatitis $C$ virus core protein residue phenylalanine 164 increases steatosis in an in vitro cellular model. Gut 2007 online (sous presse).

\title{
NOUVELLE
}

\section{Quand les polynucléaires neutrophiles attrapent les agents pathogènes dans leurs filets}

Marie-Anne Gougerot-Pocidalo, Jamel $\varepsilon l$ Benna, Pham My-Chan Dang, Carole Elbim

\author{
Inserm U773, Équipe Phagocytes, NADPH oxydases \\ et Inflammation, Faculté de Médecine, site Bichat, \\ F-75018, Paris, France ; Université Paris 7 Denis Diderot, \\ Faculté de Médecine, site Bichat, Paris, F-75018, France. \\ pocidalo@bichat.inserm.fr
}

> Les polynucléaires neutrophiles humains (PN) constituent l'un des plus puissants systèmes de défense de l'homme contre les agents pathogènes, bactéries et levures, qui ont franchi la barrière cutanéo-muqueuse. Schématiquement, sous l'influence de différents chimioattractants, les PN migrent vers leurs cibles, puis y adhèrent, les englobent et les tuent [1]. Jusqu'à présent, deux mécanismes bactéricides agissant en coopération pouvaient être distingués, I'un dépendant de l'oxygène et l'autre indépendant de celui-ci. (1) Le système tueur indépendant de l'oxygène provient essentiellement du contenu des granulations du neutrophile. Le polynucléaire neutrophile possède en effet au moins trois types de granulations cytoplasmiques: les granulations primaires ou azurophiles, les granulations secondaires ou spécifiques et les vésicules sécrétoires [2]. Lors de la formation du phagosome, les granulations fusionnent avec ce dernier et y déversent de nombreuses substances lytiques et toxiques telles que des hydrolases acides, des défensines ${ }^{1}$, la BPI (bactericidal-perme-

${ }^{1}$ Ce sont des peptides antimicrobiens de nature cationique, non glycosylés et amphiphiliques de 12 à 50 acides aminés. Elles exercent des propriétés antibactériennes, antifongiques et antivirales. Elles sont produites par plusieurs cellules principalement les neutrophiles, les cellules intestinales de Paneth, et les cellules épithéliales. ability increasing protein), l'azurocidine, le lysozyme, la lactoferrine, différentes protéases (élastase, cathepsine G...) qui vont contribuer à la destruction de l'agent pathogène englobé. (2) Le système bactéricide oxygéno-dépendant du polynucléaire neutrophile est puissant et brutal : c'est l'explosion oxydative. Elle est caractérisée par l'activation d'un système moléculaire complexe, la NADPH oxydase, qui induit une réduction de l'oxygène moléculaire en anion superoxyde $\left(\mathrm{O}_{2}^{-} \cdot{ }^{-}\right)$à l'origine d'autres formes réactives de l'oxygène telles que le peroxyde d'hydrogène $\left(\mathrm{H}_{2} \mathrm{O}_{2}\right)$, le radical hydroxyle $\left(\mathrm{OH}^{\circ}\right)$ et l'acide hypochloreux $(\mathrm{HOCl})[3,4]$.

\section{Les filets tueurs des polynucléaires}

Récemment, Brinkmann et al. [5] ont décrit un nouveau mécanisme bactéricide mis en œuvre par les PN : des PN activés par différents stimulus (cytokines, lipopolysaccharide, phorbol myristate acétate) sont capables de former des filaments extracellulaires composés d'ADN, d'histones et de protéines comme l'élastase, la cathepsine $G$, la lactoferrine et la gélatinase, issues des granulations primaires et secondaires (Figure 1 et 2). Les auteurs ont montré que ces filaments appelés NET (neutrophil extracellular traps), ou encore «filets» se lient aux bactéries à Gram positif et à Gram négatif, les piègent et exercent une activité bactéricide extracellulaire à distance sans même les englober (Figure I et 2). Les auteurs montrent que l'élastase localisée au niveau de ces NET dégrade les facteurs de virulence des bactéries pathogènes. La destruction des NET par la désoxyribonucléase, enzyme qui dégrade I'ADN, inhibe leur pouvoir bactéricide. Ces résultats sont interprétés comme un mécanisme de défense innée, indépendant de la phagocytose, qui prévient la dissémination bactérienne ou fungique, permet d'établir des concentrations importantes de substances microbicides dans le voisinage des $\mathrm{PN}$ et de tuer les agents pathogènes. Ce travail n'exclut pas que la formation de ces filets soit une étape très précoce de la mort cellulaire des PN : il est en effet généralement admis que la demi-vie courte de ces derniers est due à une mort rapide soit par nécrose soit par apoptose, selon les conditions de stimulation.

\section{Activité bactéricide}

posthume des polynucléaires

Cette même équipe vient de publier que l'émission de ces NET est associée à la 
survenue d'une mort cellulaire et les auteurs la décrivent comme différente de la mort par nécrose ou apoptose [6]. Après activation par le phorbol myristate acétate, un activateur de la signalisation intracellulaire, les PN sont observés par vidéomicroscopie : les auteurs décrivent une perte des lobes nucléaires, une homogénéisation de la chromatine, une désintégration des membranes nucléaires et granulaires permettant la mise

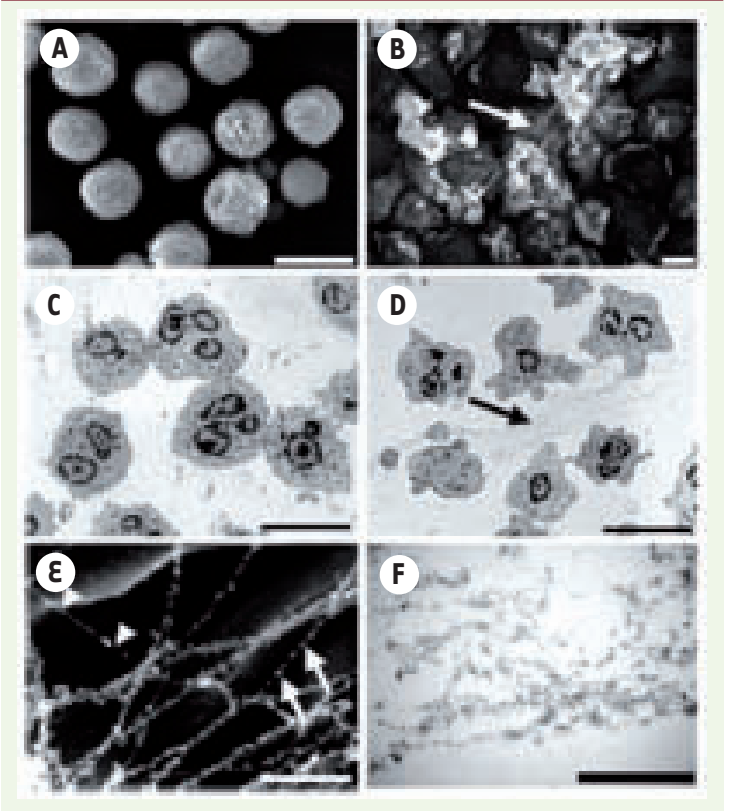

Figure 1. Analyse de PN au repos et stimulés par microscopie électronique à balayage et à transmission. $A, C$. PN au repos. $B, D$. PN stimulés; les flèches correspondent aux NET. $\varepsilon$. NET visualisés par microscopie à balayage en haute résolution. $F$. NET en sections ultrafines (d'après [4], figure I A-F publiée avec l'autorisation de Science).
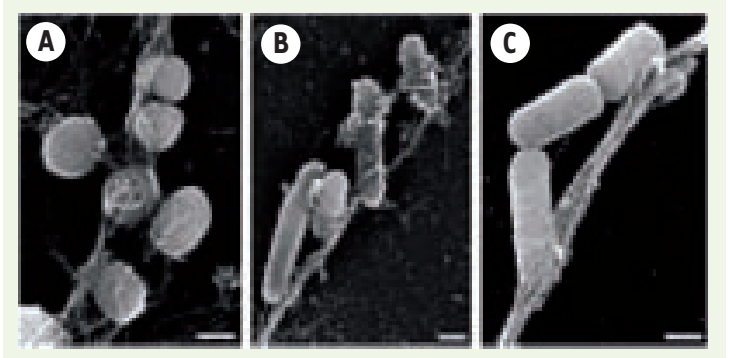

Figure 2. Formation de filets par les polynucléaires. Après stimulation par de I'IL-8, 40 minutes, les PN émettent des NET qui lient les bactéries ajoutées dans un deuxième temps. A. S. aureus. B. S. typhimurium. C. S. flexneri (d'après [4], figure 3 A-C publiée avec l'autorisation de Science). en contact des composants formant les NET et finalement leur extrusion dans le milieu extracellulaire après la rupture de la membrane plasmique. La formation des NET est dépendante des formes DPH oxydase est nécessaire à ce absence d'activité NADPH oxydase d'origine génétique (granulomatose septique chronique) [3-7] ne sont pas capables de former les NET. II est bien connu que les ROS (reactive oxygen species) jouent un rôle majeur dans la destruction intraphagocytaire des microorganismes et qu'ils induisent également la mort des PN eux-mêmes [8-10]. Ce travail montre de façon très intéressante que les PN continueraient à exercer leur activité bactéricide, même après leur mort, par l'intermédiaire des NET. La production de ROS par la NADPH oxydase active interviendrait dans la destruction des agents pathogènes à la fois directement dans le phagosome, mais aussi par l'induction d'une mort cellulaire à l'origine des NET. Cette description a mis en évidence un phénomène secondaire à la mort des PN, la formation des $\mathrm{N} \varepsilon T$, qui n'avait pas été observé dans les différentes études précédentes. Ce qui est moins clair, c'est la nouveauté du processus de mort cellulaire induit par les ROS. La description morphologique poussée s'accompagne de peu de données moléculaires permettant réellement d'affirmer qu'il s'agisse d'un nouveau processus de mort cellulaire programmée du PN induit par les ROS. Les NET ont été observés in vivo par d'autres auteurs que l'équipe de Zychlinsky, chez I'homme en situation de prééclampsie [11] et dans différents modèles animaux d'infection. Un article très récent [12] montre que le plasma de patients atteints de sepsis sévère induit I'interaction entre les plaquettes et les PN conduisant à la formation de NET piégeant les bactéries. Si le nouveau mode de défense du PN est très intéressant dans la compréhension de son activité antibactérienne et antifungique, on pourrait aussi envisager que l'exposition prolongée d'ADN de l'hôte, associé à des bactéries, puisse éventuellement participer à l'induction de maladies auto-immunes. $\diamond$

Pathogens trapped in neutrophils nets

\section{RÉFÉRENCES}

1. Witko-Sarsat V, Rieu P, Descamps-Latscha B, et al. Neutrophils: molecules, functions and pathophysiological aspects. Lab Invest 2000 ; $80: 617-53$.

2. Borregaard N, Cowland JB. Granules of the human neutrophilic polymorphonuclear leukocyte. Blood $1997 ; 89$ : 3503-21.

3. Stasia MJ. La granulomatose septique chronique $\mathrm{X}^{+}$: un fabuleux modèle d'étude de l'activation du complexe NADPH oxydase. Med Sci (Paris) 2007; 23 : 526-32.

4. Babior BM. NADPH oxidase. Curr Opin Immunol 2004 ; $16: 42-7$.

5. Brinkmann V, Reichard U, Goosmann C, et al. Neutrophil extracellular traps kill bacteria. Science $2004 ; 303$ : 1532-5

6. Fuchs TA, Abed U, Goosmann C, et al. Novel cell death program leads to neutrophil extracellular traps. J Cell Biol 2007 ; 176 : 231-41.

7. Heyworth PG, Cross AR, Curnutte JT. Chronic granulomatous disease. Curr Opin Immunol 2003; $15: 578-84$

8. Lundqvist-Gustafsson H, Bengtsson T. Activation of the granule pool of the NADPH oxidase accelerates apoptosis in human neutrophils. J Leukoc Biol 1999; 65 : 196-204.

9. Zhang B, Hirahashi J, Cullere X, Mayadas TN. Elucidation of molecular events leading to neutrophil apoptosis following phagocytosis: cross-talk between caspase 8 , reactive oxygen species, and MAPK/ERK activation. J Biol Chem 2003; 278 : 28443-54.

10. Blomgran R, Zheng L, Stendahl 0. Cathepsin-cleaved Bid promotes apoptosis in human neutrophils via oxidative stress-induced lysosomal membrane permeabilization. J Leukoc Biol 2007 online.

11. Gupta AK, Hasler P, Holzgreve W, et al. Induction of neutrophil extracellular DNA lattices by placental microparticles and IL-8 and their presence in preeclampsia. Hum Immunol 2005 ; 66 : 1146-54.

12. Urban C, Zychlinsky A. Netting bacteria in sepsis. Nat Med $2007 ; 13: 403-4$. 